\title{
Genetic Algorithm Based Hybrid Fuzzy System for Assessing Morningness
}

\author{
Animesh Biswas ${ }^{1}$ and Debasish Majumder ${ }^{2}$ \\ ${ }^{1}$ Department of Mathematics, University of Kalyani, Kalyani 741235, India \\ ${ }^{2}$ Department of Mathematics, JIS College of Engineering, Kalyani 741235, India \\ Correspondence should be addressed to Animesh Biswas; abiswaskln@rediffmail.com
}

Received 15 February 2014; Accepted 6 May 2014; Published 15 June 2014

Academic Editor: Rustom M. Mamlook

Copyright (c) 2014 A. Biswas and D. Majumder. This is an open access article distributed under the Creative Commons Attribution License, which permits unrestricted use, distribution, and reproduction in any medium, provided the original work is properly cited.

\begin{abstract}
This paper describes a real life case example on the assessment process of morningness of individuals using genetic algorithm based hybrid fuzzy system. It is observed that physical and mental performance of human beings in different time slots of a day are majorly influenced by morningness orientation of those individuals. To measure the morningness of people various self-reported questionnaires were developed by different researchers in the past. Among them reduced version of Morningness-Eveningness Questionnaire is mostly accepted. Almost all of the linguistic terms used in questionnaires are fuzzily defined. So, assessing them in crisp environments with their responses does not seem to be justifiable. Fuzzy approach based research works for assessing morningness of people are very few in the literature. In this paper, genetic algorithm is used to tune the parameters of a Mamdani fuzzy inference model to minimize error with their predicted outputs for assessing morningness of people.
\end{abstract}

\section{Introduction}

With the preference of people to perform activities at early hours of a day or later hours or in night, the circadian rhythm of individuals differs. This difference is known as morning versus evening orientation or more simply morningness. These individual differences have greater impact in circadian phase positions on physiological and psychological variables which are controlling them [1]. It is related to the ease of an adjustment to and tolerance of the night or rotating shifts [2] and influences athletic [3] and academic performance [4] at different times of the day. Several self-reporting questionnaires have been developed to measure diurnal type or distinction between "Larks" and "Owls" [5-8]. The assumption guiding these questionnaires is that people who express preference for activities at the extremes of the 24 hours of the day perform physiologic and cognitive tasks in accord with the preference [9]. Factors modulating circadian preference are increasing interest in researchers [10] and these questionnaire techniques have increasing importance in preselection, placement, and counseling of personnel engaged in rotating shift work or night work.

Morningness scales, especially the Horne and Ostberg's [5] Questionnaire (MEQ) is considered as the most popular and standardized for use in various countries and translated version like Posey and Ford [11] validated it in USA; Macacci and Zani [12] in Italy and Kerkhof [13] carried out a validation study of Dutch questionnaire; Ishihara et al., [14] validated in Japanese version; Benedito-Silva et al., [15] in Portuguese version; Adan and Almirall [16] in Spanish version; Sahu [17] in Bengali version in India. Finally, Adan and Almiral propose a reduced scale of MEQ (rMEQ) containing smallest possible items.

Several research works $[18,19]$ are made not only to support the reliability and validity of the more commonly used questionnaire but also to emphasize the methodological limitations that exist today and suggested some future avenues of work in order to overcome these limitations.

The linguistic terms which are used in preparation of rMEQ are imprecisely defined. As a result traditional tools 
very often remain inactive and do not provide satisfactory answers in analyzing collected data qualitatively. In this context fuzzy logic [20] seemed to be very much useful to handle the collected data in a proper justified way and identify morningness orientation in a more precise way. The study of fuzzy logic began in early 60s of the past century. In 1970s, fuzzy logic was combined with expert systems to become a fuzzy logic system that was then implemented in many industrial [21], medical [22], environmental [23], and agricultural [24] applications. But the application of fuzzy logic in the field of ergonomics especially assessing morningness of the people is yet to appear in the literature extensively.

A methodology [25] for assessing morningness of a group of people using adaptive neurofuzzy inference system (ANFIS) was developed in the recent past. Since it represents a first order Sugeno type fuzzy inference system (FIS), which is not particularly useful in classification applications, so, it is insufficient for diagnosis applications.

In this paper, genetic algorithm (GA) based Mamdani FIS (MFIS) has been proposed for assessing morningness of people. MFIS which has advantages in consequent parts is universally applicable and hence can be used for prognosis and diagnosis applications. But the problem with Mamdani model is the generation of fuzzy rule base which mostly depends on expert's own expertise. As a result, in the absence of expert's knowledge generation of fuzzy rules is a difficult task.

In this regard, clustering techniques are found very much helpful. Clustering algorithms not only are used extensively to organize and categorize data, but also are useful for data compression and model construction. Fuzzy clustering allows natural grouping of data in a large data set and provides a basis for constructing fuzzy rule-based system (FRBS). In the proposed methodology, subtractive clustering technique proposed by Chiu [26] is used for generating fuzzy rules which are then incorporated in a MFIS model. Basically, subtractive clustering principle is used to estimate the number of clusters and the initial locations of cluster centers. Each obtained cluster corresponds to a fuzzy rule. Chiu exploited subtractive clustering technique in a Takagi-Sugeno FIS, where the rule output is a linear function of inputs. But sometimes expert wants to classify the output variable in some predefined fuzzy classes. The number of classes in a data space is highly influenced by the parameter radius of neighborhood. So, identification of a suitable value of the radius of neighborhood for subtractive clustering is an important task. In this paper, radius of neighborhood for subtractive clustering is chosen so that the whole data space can be classified into three clusters and then cluster centers and corresponding spreads obtained by subtractive clustering are adopted in MFIS in order to generate fuzzy rules with fuzzy outputs. Then, parameters of the model thus obtained are tuned by using GA [27-29] in order to minimize the error between the real output and the predicted output obtained by the model.

The remaining part of this paper is organized in the following manner. Section 2 describes in brief the concept of GA based MFIS (GAMFIS). Section 3 presents the architecture of the proposed GAMFIS. In Section 4, a case study on assessing morningness of a group of people along with their simulation results is presented to verify the feasibility of this FRBS. Section 5 includes the concluding remarks of this work.

\section{The Basic Concepts of GAMFIS}

Fuzzy set theory provides a powerful framework to cope with vague or ambiguous problems and can express linguistic values and human subjective judgments of natural language. Among the several types of FIS, MFIS is the most popular, powerful, and widely used methodology in developing fuzzy models and analyzing data. The basic assumption proposed by Mamdani [30] underlying the approach to fuzzy logic control is that, in the absence of an explicitly defined model and/or clear statement of control design objectives, informal knowledge of the operation of the given model can be codified in terms of IF-THEN rules or condition action rules and also can form the basis for a linguistic control strategy. The fuzzy control theories based on fuzzy sets are used for the inputs and they are related to output fuzzy sets by some rules, which describe the heuristic knowledge about the behaviour of the system. The process of fuzzification of the inputs, evaluation of the rules, aggregation of all required rules, and making an inference is known as fuzzy control. For complete specification of MFIS, a function has to be assigned for each of the following operators:

(i) AND operator (usually T-norm) is used for the rule firing strength computation with AND'ed antecedents;

(ii) OR operator (usually T-conorm) is used for calculating firing strength of a rule with OR'ed antecedents;

(iii) implication operator (usually T-norm) is used for calculating qualified consequent membership functions based on given firing strength;

(iv) aggregate operator (usually T-conorm) is used for aggregating qualified consequent membership functions to generate an overall output membership function;

(v) defuzzification operator is used for transforming output membership functions to a crisp single output value.

In a GAMFIS, a GA evolves an MFIS by tuning fuzzy membership functions, learning fuzzy rules, or adapting the context. GA consists of three basic operations: reproduction, crossover, and mutation. Reproduction is the process where members of the population reproduced according to the relative fitness of the individuals, where the chromosomes with higher fitness have higher probabilities of having more copies in the coming generation. Crossover is usually applied to selected pairs of parents with a probability equal to a given crossover rate in order to exchange partially their information of the genes. Mutation is the occasional alteration of states at a particular string position. Mutation is essentially needed in some cases where reproduction and crossover alone are unable to offer the global optimal solution. It serves as an insurance policy which would recover the loss of a particular piece of information. After these three operations, the overall 
TABLE 1: rMEQ.

\begin{tabular}{|c|c|c|c|}
\hline $\begin{array}{l}\text { Question } \\
\text { number }\end{array}$ & Question & Option & Point \\
\hline 1 & $\begin{array}{l}\text { Considering only your own Feeling Best condition } \\
\text { rhythm, at what time would you get up if you were } \\
\text { entirely free to plan your day? }\end{array}$ & $\begin{array}{l}\text { (i) Before 6:30 a.m. } \\
\text { (ii) Between 6:31 a.m. and 7:45 a.m. } \\
\text { (iii) Between 7:46 a.m. and 9:45 a.m. } \\
\text { (iv) Between } 9.46 \text { a.m. and 11:00 a.m. } \\
\text { (v) After 11:00 a.m. }\end{array}$ & $\begin{array}{l}5 \\
4 \\
3 \\
2 \\
1\end{array}$ \\
\hline 2 & $\begin{array}{l}\text { During the first half hour after having awakened in the } \\
\text { morning, how tired do you feel? }\end{array}$ & $\begin{array}{l}\text { (i) Very tired } \\
\text { (ii) Fairly tired } \\
\text { (iii) Fairly refreshed } \\
\text { (iv) Very refreshed }\end{array}$ & $\begin{array}{l}1 \\
2 \\
3 \\
4\end{array}$ \\
\hline 3 & $\begin{array}{l}\text { At what time in the evening do you feel tired and, as a } \\
\text { result, in need of sleep? }\end{array}$ & $\begin{array}{l}\text { (i) Before 9:00 p.m. } \\
\text { (ii) Between 9:01 p.m. and 10:15 p.m. } \\
\text { (iii) Between 10:16 p.m. and 12:45 a.m. } \\
\text { (iv) Between 12:46 p.m. and 2:00 a.m. } \\
\text { (v) After 2:01 a.m. }\end{array}$ & $\begin{array}{l}5 \\
4 \\
3 \\
2 \\
1\end{array}$ \\
\hline 4 & $\begin{array}{l}\text { At what time of the day do you think you reach your } \\
\text { Feeling Best condition peak? }\end{array}$ & $\begin{array}{l}\text { (i) Between 5:01 a.m. and 8:00 a.m. } \\
\text { (ii) Between 8:01 a.m. and 10:00 a.m. } \\
\text { (iii) Between 10:01 a.m. and 5:00 p.m. } \\
\text { (iv) Between 5:01 p.m. and 10:00 p.m. } \\
\text { (v) Between 10:01 p.m. and 5:00 a.m. }\end{array}$ & $\begin{array}{l}5 \\
4 \\
3 \\
2 \\
1\end{array}$ \\
\hline 5 & $\begin{array}{l}\text { One hears about morning and evening types of people. } \\
\text { Which one of these types do you consider yourself to } \\
\text { be? }\end{array}$ & $\begin{array}{l}\text { (i) Definitely a morning type } \\
\text { (ii) Rather morning than evening } \\
\text { (iii) Rather evening than morning } \\
\text { (iv) Definitely an evening type }\end{array}$ & $\begin{array}{l}6 \\
4 \\
2 \\
0\end{array}$ \\
\hline
\end{tabular}

fitness of the population improves. Each population generated then goes through a series of evaluation, reproduction, crossover, and mutation, and the procedure is repeated until the termination condition is reached.

\section{Methodological Developments}

To develop the proposed methodology, the set of five questions in rMEQ is considered in Table 1.

To quantify the collected data on the basis of expert's opinion, a preference scale is adopted on the basis of responses by the subjects.

It is difficult for a person to express his Feeling Best condition at a specific time of a day or it is troublesome to measure the tiredness during the first half hour after waking up in the morning in a precise way, due to fuzziness in human behaviours. So, the fuzziness is inherently involved with every question of the questionnaire. An FIS employing fuzzy IFTHEN rules can only model the qualitative aspects of human knowledge and reasoning processes without employing any precise quantitative analysis.

Once the responses of the group of people are obtained, the tasks in the design phase are the selection of fuzzy input and output parameters for the model, partitioning of inputoutput data space and developing a knowledge base, selection of suitable implication, aggregation, and defuzzification operators, and tuning of parameters of the MFIS by GA.

3.1. Selection of Fuzzy Input and Output Parameters. The parameters which are considered in this study as fuzzy inputs are average rising time for Feeling Best condition $\left(X_{1}\right)$, feelings within the first half hour after waking up in the morning $\left(X_{2}\right)$, sleeping time $\left(X_{3}\right)$, Feeling Best time in a day $\left(X_{4}\right)$, and self-assessment about morningness $\left(X_{5}\right)$ and morningness $(Z)$ is considered as fuzzy output parameter.

3.2. Use of Subtractive Clustering for the Development of Knowledge Base System. The main part of the MFIS is the construction of knowledge base system which consists of data base and rule base. The data base defines the membership functions of fuzzy sets used in the fuzzy rules, whereas rule base contains a number of fuzzy IF-THEN rules. Clustering of numerical data forms the basis of many classification and system modeling algorithms. The purpose of clustering is to distill natural groupings of data from a large data set, producing a concise representation of a system's behavior. When the cluster estimation method is applied to a collection of input/output data, each cluster center is in essence a prototypical data point that exemplifies a characteristic behavior of the system. Hence, each cluster center can be used as the basis of a rule that describes the system behavior. For identification of Mamdani fuzzy model, the subtractive clustering estimation method is used here as the basis of a fast and robust algorithm.

Three fuzzy sets, namely, morning $(M)$, intermediate $(I)$, and evening $(E)$, are considered to express the output variable morningness $(Z)$. So, the output variable would have to classify in three predefined fuzzy classes. As the number of classes in a data space obtained by subtractive clustering is highly influenced by the parameter radius of neighborhood $(r)$, a value is assigned to the parameter as radius of neighborhood $(r)$ in such a way that subtractive clustering produces three 


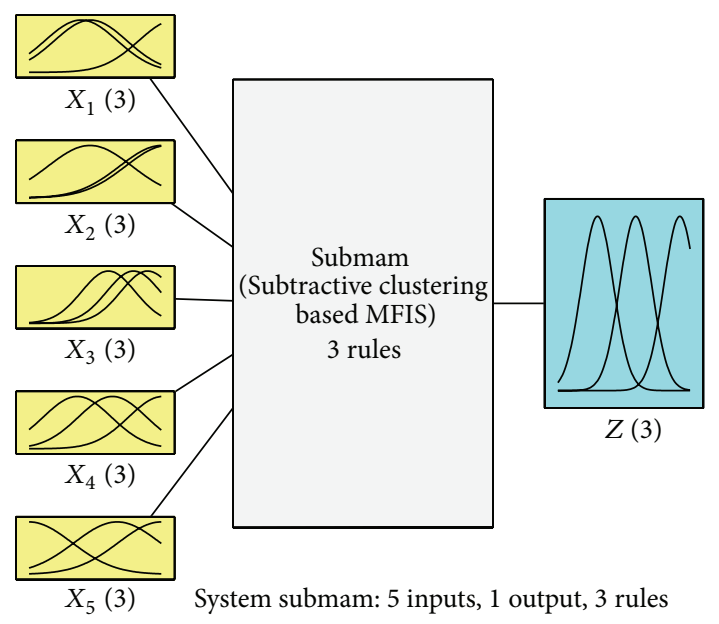

Figure 1: Mamdani FIS.

clusters in the input-output data space. The cluster centers and corresponding spreads obtained by subtractive clustering are represented in matrix form as follows:

$$
\begin{gathered}
C=\left[\begin{array}{llllll}
c_{11} & c_{12} & c_{13} & c_{14} & c_{15} & c_{16} \\
c_{21} & c_{22} & c_{23} & c_{24} & c_{25} & c_{26} \\
c_{31} & c_{32} & c_{33} & c_{34} & c_{35} & c_{36}
\end{array}\right] ; \\
S=\left[\begin{array}{llllll}
s_{1} & s_{2} & s_{3} & s_{4} & s_{5} & s_{6}
\end{array}\right] .
\end{gathered}
$$

These cluster centers and spreads have been exploited in a MFIS model with three fuzzy rules of the following form.

$R_{i}$ : If input 1 is close to $c_{i 1}$ and input 2 is close to $c_{i 2}$ and........ and input 5 is close to $c_{i 5}$ then the output is close to $c_{i 6}$ for $i=1,2,3$.

The most commonly used Gaussian type membership functions are considered here for both premises and consequent parameters of each of the three fuzzy rules (see Figure 1).

Here, $c_{i j}(i=1,2,3 ; j=1,2, \ldots, 6)$ and $s_{j}(j=1,2, \ldots, 6)$ are used as centers and width, respectively, of three Gaussian type fuzzy sets corresponding to the $j$ th variable.

3.3. Implication, Aggregation, and Defuzzification Operators Selection. In this paper, Mamdani model, in which three fuzzy rules are injected, is used with the following operators:

(i) min operator for conjunction;

(ii) max operator for union;

(iii) min operator for implication;

(iv) max operator for aggregation;

(v) centre of area (COA) operator for defuzzification.

3.4. Tuning of MFIS Parameters by GA. Here, since Gaussian type membership functions are chosen for both premises and consequent parameters of each fuzzy rule, the Mamdani model thus obtained contains the Gaussian membership functions with a center and a width for each of the inputs and outputs. Thus, the total number of parameters is equal to number of membership functions $\times$ number of variables + number of variables; that is, there are a total 24 parameters which are involved in the proposed Mamdani model. The next task is to tune all parameters of the Mamdani model using GA in order to minimize the error between the real output $y_{k}$ and the predicted output $y(k)$ obtained by the proposed methodology. Here, the root mean squared error (RMSE) between the two outputs for the training data set is chosen as objective function which is defined as

$$
\mathrm{RMSE}=\sqrt{\frac{1}{n} \sum_{k=1}^{n}\left(y(k)-y_{k}\right)^{2}},
$$

where $n$ is the number of training data.

Each step of the tuning process is illustrated and detailed as follows.

Step 1. The first step is to create an initial population of possible models or chromosomes. The population of chromosomes is generally chosen randomly. There are no hard rules for determining the size of the population. Generally, the size is defined to range between 20 and 100. Large populations guarantee greater diversity and may produce more robust solutions. In the proposed methodology, initial populations are generated by using subtractive clustering repeatedly for different values of " $r$ " so that the number of clusters remains three in each case. As the number of classes in a data space obtained by subtractive clustering is highly influenced by radius of neighborhood $(r)$, the lower bound (LB) and upper bound (UB) for the value of radius of neighborhood $(r)$ are identified in such a way that the number of clusters for the input-output data set becomes three. To determine the LB and UB within which every value of " $r$ " subtractive clustering results in three clusters, an M-code is developed for MATLAB.

Now, for any value of " $r$ " within the interval [LB, UB] subtractive clustering will produce three clusters in the inputoutput data space whose cluster centers and corresponding spreads are obtained in the following form:

$$
\begin{gathered}
C=\left[\begin{array}{llllll}
c_{11} & c_{12} & c_{13} & c_{14} & c_{15} & c_{16} \\
c_{11} & c_{22} & c_{23} & c_{24} & c_{25} & c_{26} \\
c_{31} & c_{32} & c_{33} & c_{34} & c_{35} & c_{36}
\end{array}\right] ; \\
S=\left[\begin{array}{llllll}
s_{1} & s_{2} & s_{3} & s_{4} & s_{5} & s_{6}
\end{array}\right] .
\end{gathered}
$$

Varying the value of " $r$ " within the interval [LB, UB], different sets of cluster centers and corresponding spreads are obtained which will together constitute the initial population for GA.

Step 2. The assignment of the fitness in GA serves as a guidance to lead the optimal solution. Hence, a fitness function composed of RMSE is proposed in this method to compare the possible models performance or prediction accuracy. The closer the value of RMSE to 0 is, the better the forecasting accuracy is. Hence, to improve the accuracy of the results, RMSE has to be minimized. 


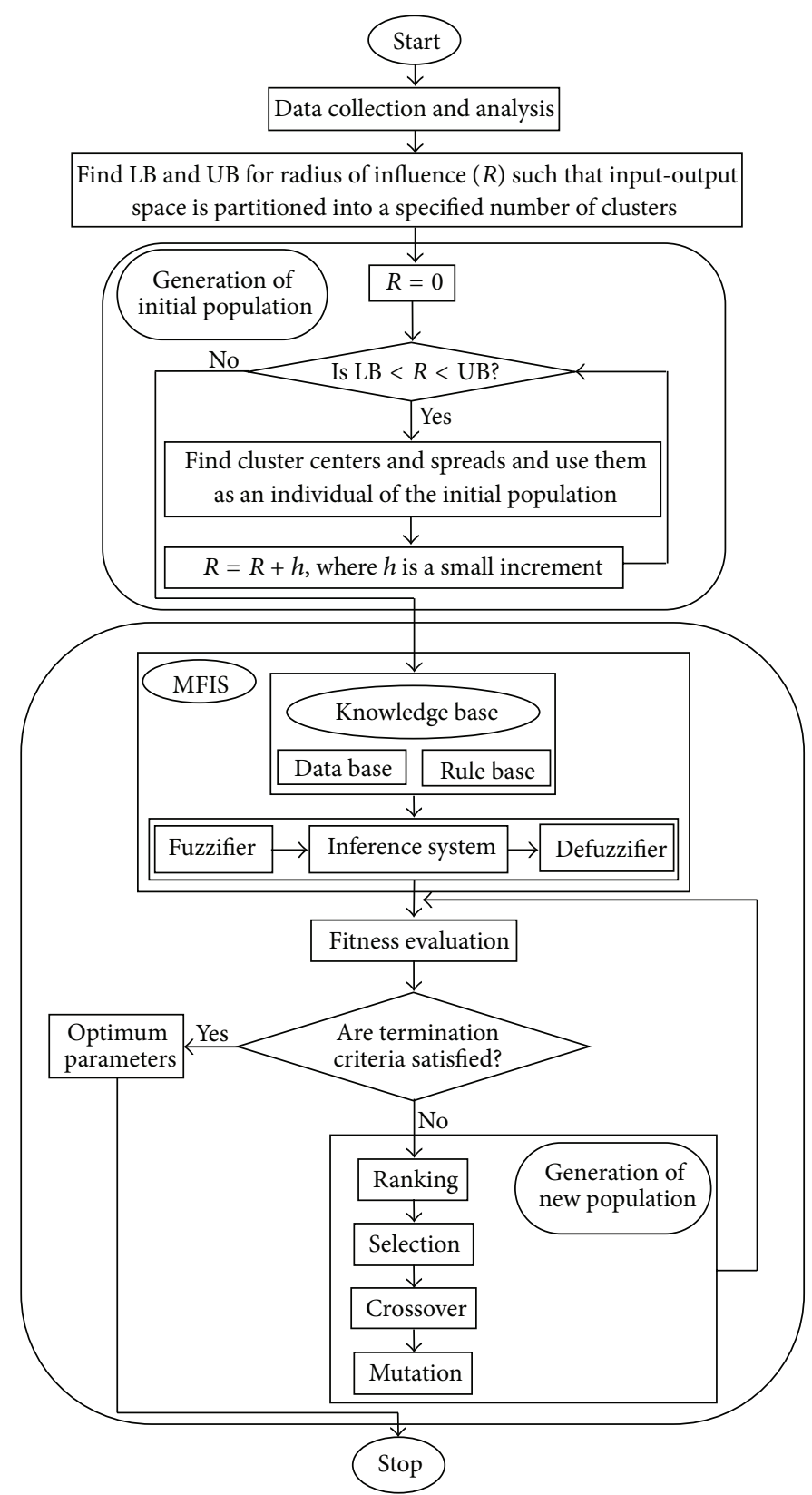

FIGURE 2: Flowchart for the suggested method.

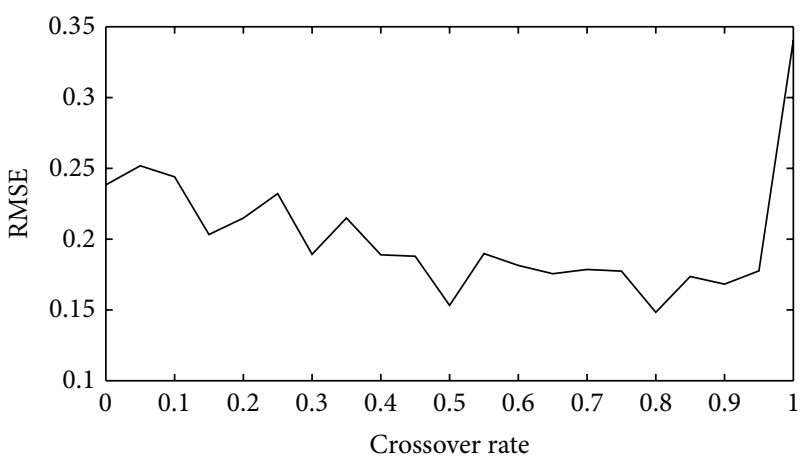

Figure 3: Crossover rate versus RMSE.
Step 3 (selection and mutation for all chromosomes in the mating pool). After each of the chromosomes is evaluated and associated with fitness, the current population undergoes the reproduction process to create the next generation of population. The reproduction process is the combination of three types of operations.

(i) Selection of elite children which are the individuals in the current generation with the best fitness values: these individuals automatically survive to the next generation.

The "Roulette wheel" selection scheme is used here to determine the members of the new generation population. After the selection (reproduction) process, the 
population is enriched with better individuals. Reproduction makes clones of good strings but does not create new ones.

(ii) Crossover operator is applied to the mating pool with the hope that it creates a better offspring. In this method, single point crossover is used to combine the vectors of a pair of parents.

(iii) After crossover, the strings are subjected to mutation. Mutation prevents the algorithm from being trapped in a local minimum. Mutation plays the role of recovering the lost genetic materials and randomly disturbing genetic information. Mutation children are created by introducing random changes or mutations to a single parent. There are many different forms of mutation for the different kinds of representation.

After these three operations, the overall fitness of the population is improved.

Step 4. Replace the current population with the offspring to form the next generation.

Step 5. If the end conditions are not satisfied, go to Step 2. Otherwise, stop and retain the best solution in current population.

The proposed methodology, GAMFIS, is schematically shown in Figure 2.

\section{Morningness Assessment of a Group of Students}

To illustrate the potentiality of the proposed morningness assessment process, an illustrative case example on assessing morningness of students at University of Kalyani, West Bengal, India, is presented. The sample of 530 postgraduate students is randomly taken from the university to collect their responses corresponding to each item of the rMEQ questionnaire on voluntary basis. Almost fifty percent of them are residential students and fifty percent are day boarders. All of them are acquainted with English. After describing details of the study, the English version of rMEQ was handed over to them. They filled up the questionnaire and returned it. The responses are quantified using the preference scale developed for this purpose. In this paper, whole data set is divided into two subsets. The first subset is the training set ( $~ 85 \%$ of whole data) by which an input-output relationship has been obtained. The second subset is testing set ( $\sim 15 \%$ of whole data).

In the context of assessing morningness, three fuzzy sets morning $(M)$, intermediate $(I)$, and evening $(E)$ are considered for the output variable morningness. So, the output variable is classified in three predefined fuzzy classes. Here, inputoutput data space has been partitioned into three clusters using subtractive clustering and the cluster centers and corresponding spreads thus obtained are used to develop MFIS. Using M-code written in MATLAB, the LB and UB of " $r$ " for which the subtractive clustering partitioned the input-output data space formed by the collected data into three clusters are
TABLE 2: Criteria for termination of GA.

\begin{tabular}{lc}
\hline Options & Limits \\
\hline Generations & 100 \\
Time limit & Inf \\
Fitness limit & 0 \\
Stall generations limit & 50 \\
Stall time limit & Inf \\
Function tolerance & $1 \mathrm{e}^{-6}$ \\
\hline
\end{tabular}

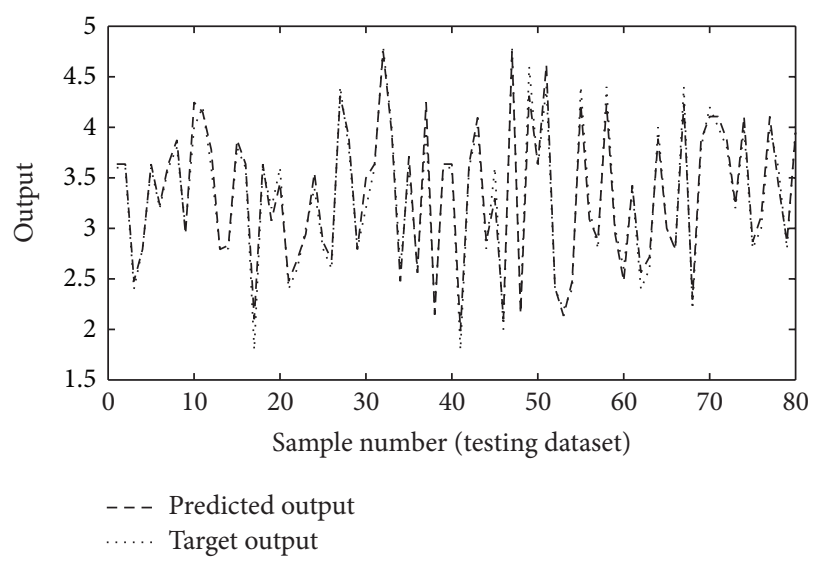

FIgURE 4: Comparison between target output and predicted output.

obtained as 0.882 and 1.279 , respectively. Now, varying the value of " $r$ " from 0.882 to 1.279 with an increment 0.001 an initial population of size 398 has been formed in which each individual represents the cluster centers and corresponding spreads for each of those specific values of " $r$."

After each of the individuals of a generation is evaluated and associated with fitness, the current population undergoes the reproduction process to create the next generation of population. Here the "Roulette wheel" selection scheme is used to determine the member of the new generation population. Once the new generation is obtained the mating pool has been formed and the crossover is carried out with a specific rate between 0 and 1 . Since, crossover rate is one of the most influential parameters in GA, the whole process is performed for different values of crossover rate ranging between 0 and 1 with an increment of 0.05 . Thus, a total of 21 experiments are performed to find the best value of crossover rate. Figure 3 shows that optimum value of the fitness function (RMSE) has been attained corresponding to the crossover rate of 0.8 . Thus, GA has been performed with crossover rate 0.8 which is then followed by the mutation operation. GA has been run with the termination criteria as given in the Table 2.

4.1. Results and Discussions. All procedures are implemented with MATLAB R2008a. After the termination of the whole process the best cluster centers and the corresponding spreads (as given by Table 3 ) thus obtained are used to get the optimum MFIS for assessing morningness of an individual. 
TABLE 3: Best cluster centers and corresponding spreads.

\begin{tabular}{|c|c|c|c|c|c|c|}
\hline Cluster centers and spreads & Input 1 & Input 2 & Input 3 & Input 4 & Input 5 & Output \\
\hline 1st Cluster & 2.3281 & 4.0110 & 3.9515 & 3.1545 & 4.0000 & 3.5337 \\
\hline 2nd Cluster & 5.5204 & 4.1920 & 4.4833 & 5.0524 & 6.0000 & 5.5340 \\
\hline 3rd Cluster & 1.9675 & 1.8443 & 3.0000 & 1.8242 & 0 & 1.7999 \\
\hline Spreads & 1.3674 & 1.2677 & 1.0140 & 1.2875 & 1.0426 & 0.7237 \\
\hline
\end{tabular}

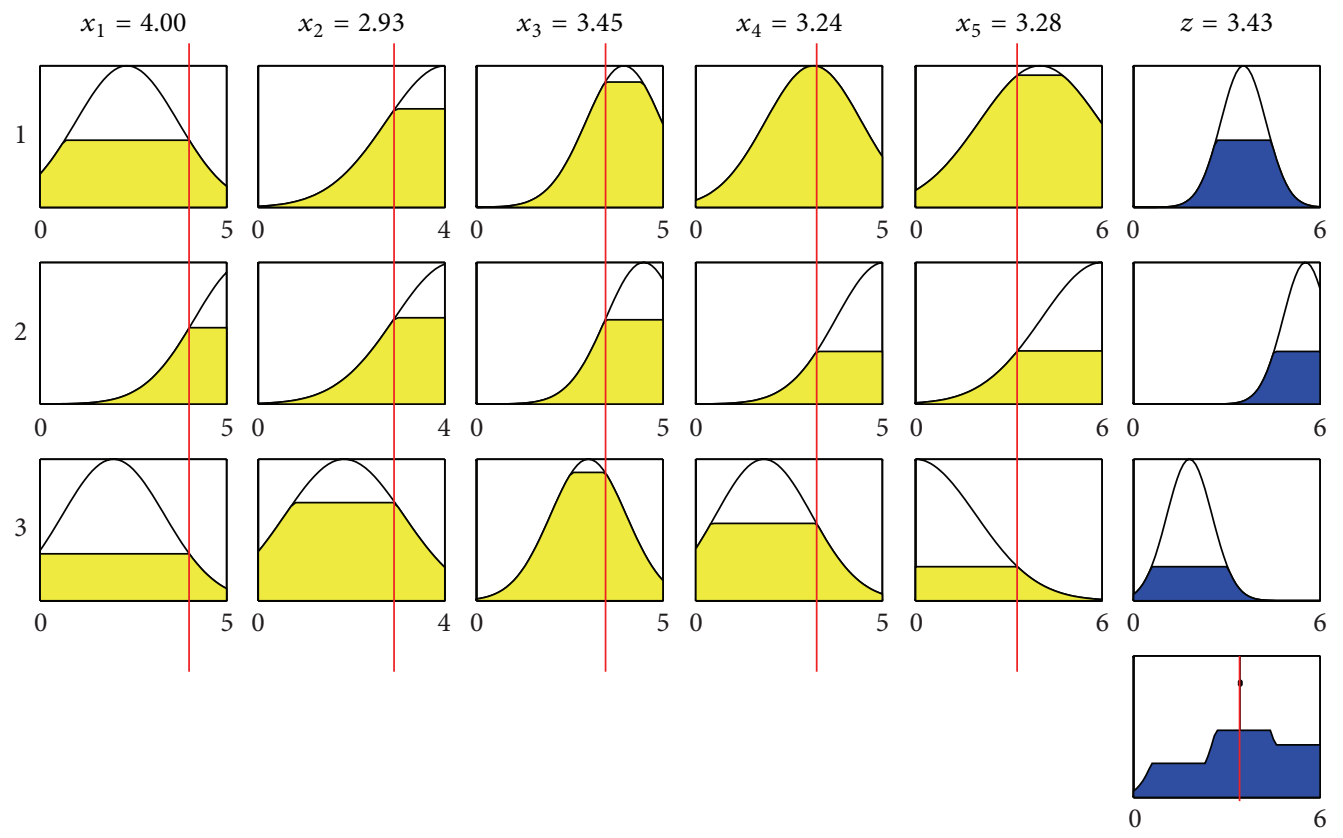

FIgURE 5: Diagram of GAMFIS rules and output.

The RMSE between the real and predicted outputs after adjustment with GA is 0.1529 . Figure 4 presents a comparison between target output and predicted output.

Thus a Mamdani fuzzy model tuned by GA is developed for assessing morningness of people and it is found that the RMSE is small. On the basis of the developed model degrees of morningness of any individual can be measured if he/she provides the input through the rMEQ questionnaire. In this context, if the average preference scale values of the responses made by the university students are considered, then the values corresponding to five different linguistic variables are found as $X_{1}=4.00, X_{2}=2.93, X_{3}=3.45, X_{4}=3.24$, and $X_{5}=3.28$.

Then the assessment through the newly generated GA based Mamdani model is found as $Z=3.43$ (Figure 5).

The membership value of morningness through the defined output fuzzy set corresponding to $Z=3.43$ is calculated as $\mu_{M}(z)=0.143, \mu_{I}(z)=0.785$, and $\mu_{E}(z)=0$ (Figure 6).

The result obtained by proceeding through this process reflects that the group of students is "intermediate" type with membership value of 0.785 and "morning" type with membership value of 0.143 .

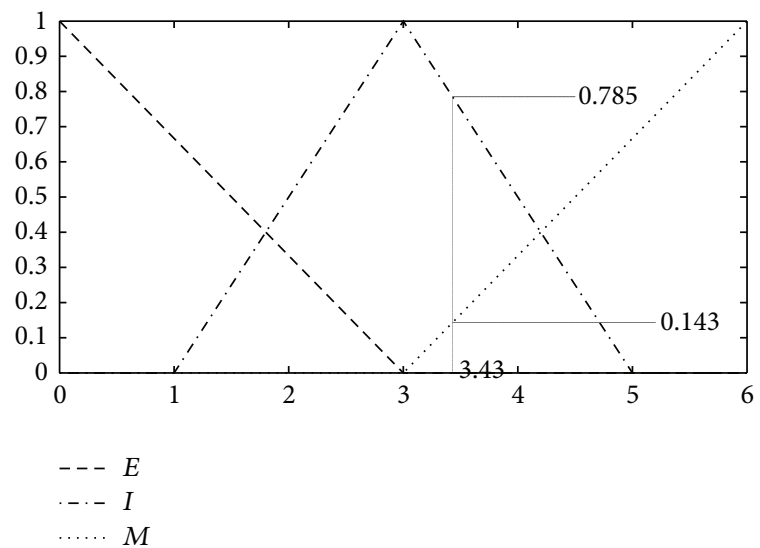

Figure 6: Measurement of the degree of morningness.

\section{Conclusions}

A morningness assessment model based on MFIS is developed through this paper which is then tuned by GA in order to minimize the error between the real and predicted output 
obtained by the fuzzy model. Here, GA is performed for various values of crossover rate and after sensitivity analysis the optimal value is identified which is then used for final execution of GA. The importance of this study is that on the basis of the morningness assessment of an individual by this proposed model an individual can be categorized more precisely as $M, I$, or $E$ type with some membership grade. The higher grade for any of the abovementioned fuzzy sets implies the greater acceptability of the corresponding type in the assessment process of that individual. This precise membership grade may be utilized in predicting more productive time period of an individual and suitability for different shifts.

\section{Conflict of Interests}

The authors declare that there is no conflict of interests regarding the publication of this paper.

\section{Acknowledgments}

The authors are thankful to the anonymous reviewers for their valuable comments and suggestions to improve the quality of the paper. The authors wish to convey deep gratitude to all the subjects who volunteered for the study and gladly gave consent. The authors are very much grateful to Dr. S. Sahu, Assistant Professor, Department of Physiology, University of Kalyani, Kalyani, India, for his kind support in collecting the data and also for his expert suggestions in developing the model.

\section{References}

[1] G. M. Cavallera and S. Giudici, "Morningness and eveningness personality: a survey in literature from 1995 up till 2006," Personality and Individual Differences, vol. 44, no. 1, pp. 3-21, 2008.

[2] R. Moog, "Optimization of shift work: physiological contributions," Ergonomics, vol. 30, no. 9, pp. 1249-1259, 1987.

[3] R. S. Smith, C. Guilleminault, and B. Efron, "Circadian rhythms and enhanced athletic performance in the national football league," Sleep, vol. 20, no. 5, pp. 362-365, 1997.

[4] R. J. Callan, "The potential effects of chronobiology on taking Scholastic aptitude test," Clearing House, vol. 68, pp. 174-176, 1995.

[5] J. A. Horne and O. Ostberg, "A self assessment questionnaire to determine Morningness Eveningness in human circadian rhythms," International Journal of Chronobiology, vol. 4, no. 2, pp. 97-110, 1976.

[6] L. Torsvall and T. Akerstedt, "A diurnal type scale construction, consistency and validation in shift work," Scandinavian Journal of Work, Environment and Health, vol. 6, no. 4, pp. 283-290, 1980.

[7] R. Moog, "Morningness- evening types and shift work a questionnaire study," in Night and Shift Work: Biological and Social Aspects, A. Reinberg, N. Vieux, and P. C. Andlaner, Eds., pp. 481-488, Pergamon Press, Oxford, UK, 1981.

[8] C. S. Smith, C. Reilly, and K. Midkiff, "Evaluation of three circadian rhythm questionnaires with suggestion for improved measures of 'morningness"', Journal of Applied Psychology, vol. 74, no. 5, pp. 728-738, 1989.
[9] C. S. Smith, S. Folkard, R. A. Schmieder et al., "Investigation of morning-evening orientation in six countries using the preferences scale," Personality and Individual Differences, vol. 32, no. 6, pp. 949-968, 2002.

[10] L. Tonetti, S. Sahu, and V. Natale, "Circadian preference in Italy and India: a comparative study in young adults," Personality and Individual Differences, vol. 53, no. 3, pp. 837-842, 2012.

[11] T. B. Posey and J. A. Ford, "The morningness-eveningness preference of college students as measured by the Horne and Ostberg questionnaire," International Journal of Chronobilogy, vol. 7, pp. 141-144, 1981.

[12] L. Macacci and A. Zani, "Morningness-eveningness preferences and sleep-waking diary data of morning and evening types in student and worker samples," Ergonomics, vol. 26, no. 12, pp. 1147-1153, 1983.

[13] G. A. Kerkhof, "A Dutch-language questionnaire for selection of morning and evening type individuals," Nederlands Tijdschrift voor de Psychologie, vol. 39, pp. 281-294, 1984.

[14] K. Ishihara, A. Miyashita, M. Inugami, K. Fukuda, K. Yamazaki, and Y. Miyata, "The results of investigation by the Japanese version of Morningness-Eveningness Questionnaire," The Japanese journal of psychology, vol. 57, no. 2, pp. 87-91, 1986.

[15] A. A. Benedito-Silva, I. S. Menna-Barreto, J. Cipolla-Neto, N. Marques, and S. Tenreiro, "A self-evaluation questionnaire for the determination of morningness-eveningness types in Brazil," Chronobiologia, vol. 16, no. 3, p. 311, 1989.

[16] A. Adan and H. Almirall, "Adaptation and standardization of a Spanish version of the morningness-eveningness questionnaire: Individual differences," Personality and Individual Differences, vol. 11, no. 11, pp. 1123-1130, 1990.

[17] S. Sahu, Ergonomics studies on personnel working in shifts in different health care units for improvement of efficiency, occupational health and safety [Ph.D. thesis], University of Calcutta, 1997.

[18] A. Adan, S. N. Archer, M. P. Hidalgo, L. Di Milia, V. Natale, and C. Randler, "Circadian typology: a comprehensive review," Chronobiology International, vol. 29, no. 9, pp. 1153-1175, 2012.

[19] D. Milia, A. Adan, V. Natale, and C. Randler, "Reviewing the psychometric properties of contemporary circadian typology measures," Chronobiology International, vol. 30, no. 10, pp. 12611271, 2013.

[20] L. A. Zadeh, "Fuzzy sets," Information and Control, vol. 8, no. 3, pp. 338-353, 1965.

[21] G. E. Gürcanli and U. Müngen, "An occupational safety risk analysis method at construction sites using fuzzy sets," International Journal of Industrial Ergonomics, vol. 39, no. 2, pp. 371-387, 2009.

[22] M. A. Vila and M. Delgado, "On medical diagnosis using possibility measures," Fuzzy Sets and Systems, vol. 10, no. 1-3, pp. 211-222, 1983.

[23] H. Cao and G. Chen, "Some applications of fuzzy sets to meteorological forecasting," Fuzzy Sets and Systems, vol. 9, no. 1-3, pp. 1-12, 1983.

[24] A. Biswas and B. B. Pal, "Application of fuzzy goal programming technique to land use planning in agricultural system," Omega, vol. 33, no. 5, pp. 391-398, 2005.

[25] A. Biswas, D. Majumder, and S. Sahu, "Assessing morningness of a group of people by using fuzzy expert system and adaptive neuro fuzzy inference model," Communications in Computer and Information Science, vol. 140, pp. 47-56, 2011. 
[26] S. L. Chiu, "Extracting fuzzy rules from data for function approximation and pattern classification," in Fuzzy Information Engineering: A Guided Tour of Applications, D. Dubois, H. Prade, and R. Yager, Eds., chapter 9, John Wiley \& Sons, New York, NY, USA, 1997.

[27] J. H. Holland, Adaptation in Natural and Artificial Systems, The University of Michigan Press, 1975.

[28] P. Pulkkinen, J. Hytönen, and H. Koivisto, "Developing a bioaerosol detector using hybrid genetic fuzzy systems," Engineering Applications of Artificial Intelligence, vol. 21, no. 8, pp. 13301346, 2008.

[29] P. Tahmasebi and A. Hezarkhani, "A hybrid neural networksfuzzy logic-genetic algorithm for grade estimation," Computers and Geosciences, vol. 42, pp. 18-27, 2012.

[30] E. H. Mamdani, "Advances in the linguistic synthesis of fuzzy controllers," in Fuzzy Reasoning and Applications, E. H. Mamdani and B. R. Gaines, Eds., Academic Press, London, UK, 1981. 

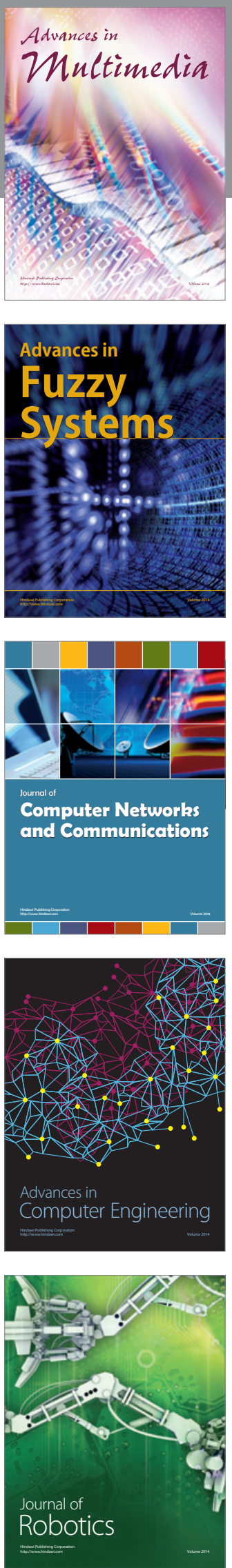

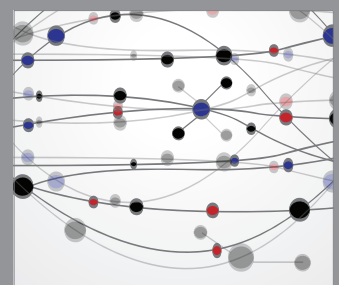

The Scientific World Journal
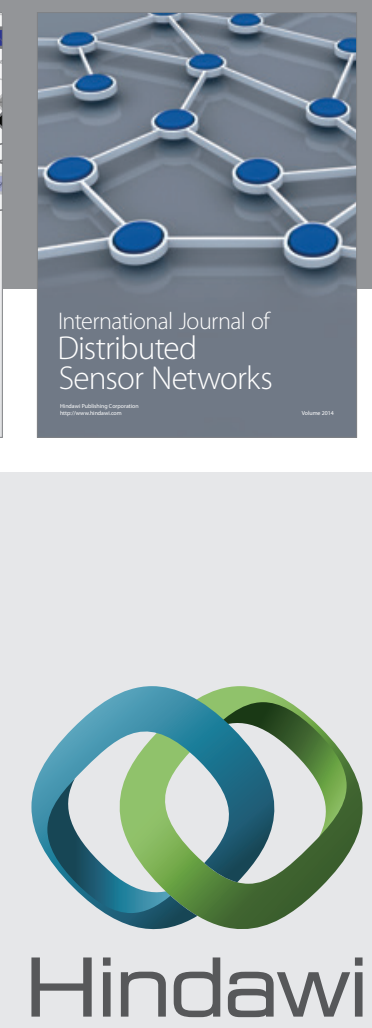

Submit your manuscripts at

http://www.hindawi.com
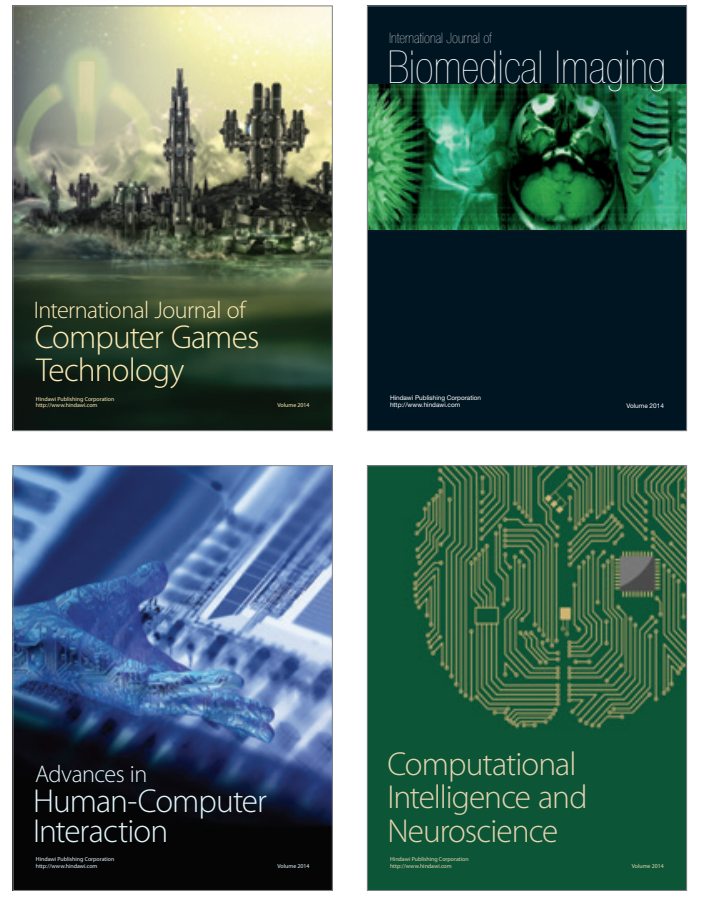
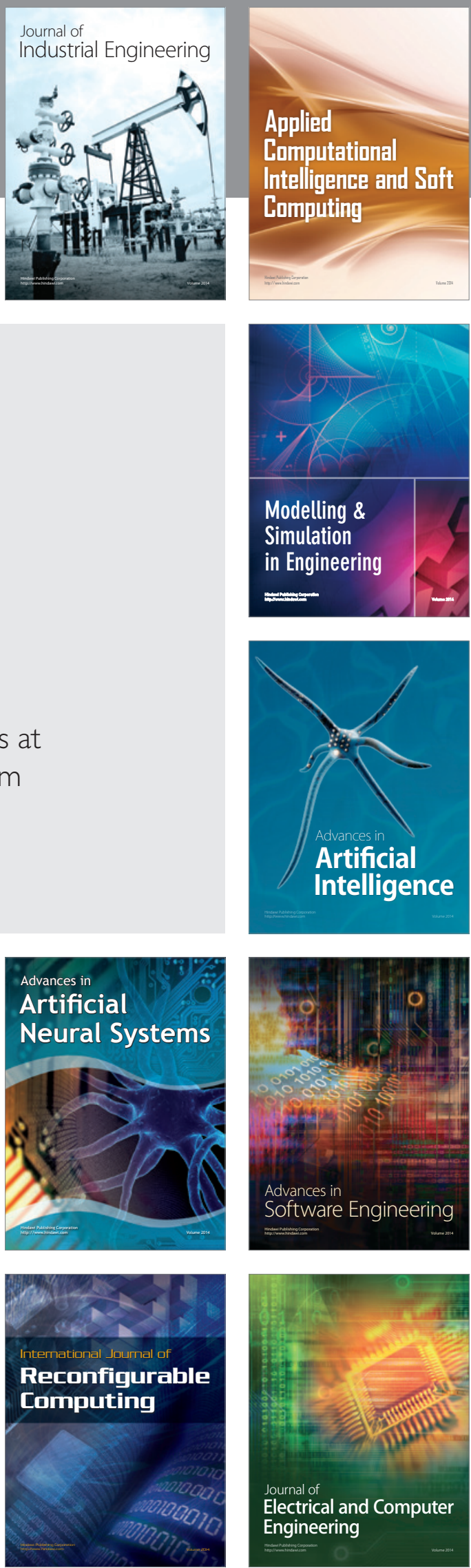